\title{
Fast Convergence Scheme for Potential-based Routing in Wireless Sensor Networks
}

\author{
Alireza Sheikhattar, Mehdi Kalantari \\ University of Maryland, College Park \\ Email: \{arsha89, mehkalan\}@umd.edu
}

\begin{abstract}
In the electrostatics-inspired methods, a potential function determines the routing mechanism for transportation of nodes' traffic. In a potential-based routing, it is essential to calculate the potential function at the location of nodes. The best existing algorithm proposed for distributed potential calculation makes use of Jacobi iterations. These iterations are well-known for their slow convergence which makes them useless for practical applications. Our proposed method aims at improving the convergence rate of potential calculation. We propose a novel method inspired by wave propagation in physical media by making analogy between a simple path network and a single stub transmission line. We generalize our method to make it applicable to all possible network topologies. Analysis results show that the required number of iterations reduces significantly to $O(N)$ for the path network of $N$ nodes, compared to $O\left(N^{2}\right)$ for Jacobi iterations.
\end{abstract}

\section{INTRODUCTION}

In recent decades, technical advancements in microelectronics, microcomputing, and communications have enabled deployment of massively dense wireless and wired networks. Wireless sensor networks and networks of machines for cloud computing are two distinguished examples of dense networks. In such networks, the number of interconnected nodes can be unboundedly large. As the number of nodes grows larger, careful analysis of the network behavior becomes computationally prohibitive by using conventional methods. Several electrostatics-inspired models have been proposed in recent years to overcome the complexity issues of dense networks. In these models, a potential function is used as the basis for routing of information flow from sources to their corresponding destinations. This potential function determines a simple routing policy such that the input flow to each node is divided proportional to the potential decent to respective neighbor nodes. In the previous work [1], the potential-based approach is used for load balancing. The potential-based routing can also be used for optimization of the information sink locations and the coverage areas of different sinks in a wireless network [2], [3]. Moreover, [4] has shown that this routing method minimizes the number of nodes required to carry a specified information density in a network area.

A major drawback of the potential-based approaches is that almost all of them assume that the potential values are calculated at a central node and then communicated to all nodes

This work is partially supported by National Science Foundation under grant 0931957. in the network. Such assumptions are prohibitive, because the potential function is a function of nodes' density as well as the geometrical distribution of sources and sinks in the network. Therefore, a centralized method for potential calculation is not practical in dynamic networks. Recent works in [5] and [6] have offered a technique based on Jacobi iterations, which calculates the potential function in a distributed (local-control) way using simple iterative computations between adjacent nodes. Such Jacobi iterations use the average potential values of neighboring nodes as the new potential value for a node. It is proved that these potential values converge to actual potentials. However, a major drawback of the proposed approach based on Jacobi iterations is that it requires too many iterations to converge. As a matter of fact, it will be shown that the number of iterations required for convergence of the Jacobi method is $O\left(N^{2}\right)$ for a path network, where $N$ is total number of nodes in the network. Hence, Jacobi iterations are not practical for distributed potential calculation in large networks.

In this paper, we propose a novel method for distributed calculation of the potential values. To accelerate the convergence rate, we use a method inspired by wave propagation in a physical transmission line. This fast convergence method is derived from partial differential equations for propagation of voltages (or currents) in physical medium. We show that the proposed method converges to the same potentials as Jacobi method by far less iterations. Indeed, we show that the iterations required for convergence of our proposed method is reduced to $O(N)$ for a path network of $N$ nodes. This significant improvement in the convergence rate is an essential step toward the practical use of potential-based methods in communication networks.

The remainder of this paper is organized as follows: Section II reviews some background work and discusses drawbacks of existing methods for potential calculation. Section III discusses our proposed method based on transmission lines. Analysis of our method is given in Section IV. Comparisons and numerical examples will be discussed in Section V. Finally, Section VI concludes the paper.

\section{Motivation}

In a potential-based routing, it is essential to compute the potential function at the location of nodes. Generally, it is desirable to calculate potential values in a distributed fashion (i.e., nodes compute their potential values through a sequence of distributed 


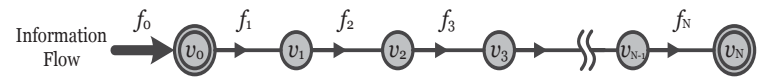

Fig. 1. A path network with source node as the first vertex $\left(w_{0}\right)$ and sink node as the last vertex $\left(v_{N}\right)$ and $(N-1)$ relay nodes

iterations with their neighbors). The best known method for distributed potential calculation uses Jacobi iterations [7]. The Jacobi iterations are expressed in the form:

$$
\mathbf{u}^{(n+1)}=\mathbf{S} \mathbf{u}^{(n)}+\mathbf{b}
$$

where $\mathbf{S}$ and $\mathbf{b}$ are the iteration matrix containing averaging weights and constant vector of bias elements, respectively. The vector $\mathbf{u}^{(n)}$ contains nodes' potentials in the $n$-th iteration which gives better approximations to the optimal solution as $n$ grows large. The iteration matrix for Jacobi method has the property that elements of each row are non zero only for the neighbors of the corresponding node. Therefore, the potential at each node is updated in a distributed fashion such that each node updates its own potential by averaging the neighboring nodes' potential and adding a bias proportional to traffic injecting at node. The distributed nature of the Jacobi method is an important factor that makes it suitable for practical networks; however, convergence rate of this method is generally very slow, which requires large number of iterations and inter-node communication. This slow convergence property causes overhead in energy consumption which reduces the lifespan of the network, particularly in case of battery powered wireless networks. As an explicit example to illustrate the slow convergence of Jacobi method, consider a simple path network with one source and sink at both ends. As shown in Figure 1, information is injected to the network at the first node, going through $(N-1)$ relay nodes to reach the sink node at the other end. An important question is that how fast potentials converge to their final values. The convergence of distributed methods rely on the eigenstructure of the network. The potential vector $\mathbf{u}^{(n)}$ converges to the optimal solution at a rate governed by the dominant eigenvalue of the iteration matrix. The general form of the iteration matrix spectrum for path network can be computed based on Laplacian spectrum in the following way:

$$
\lambda_{i}^{s}=\cos \left(\frac{(2 i-1) \pi}{2 N}\right), \quad 1 \leq i \leq N
$$

where $\lambda_{i}^{s}$ are the eigenvalues of state matrix in Jacobi iterations. The dominant eigenvalue is equal to $\lambda_{1}^{s}=\cos \left(\frac{\pi}{2 N}\right)$ and its distance to unity can be computed as follow

$$
d=2 \sin ^{2}\left(\frac{\pi}{4 N}\right)
$$

where for large $N$, it can be approximated by $d \approx \frac{\pi^{2}}{8 N^{2}}$. It can be seen that the number of required iterations for convergence is in order of $O\left(N^{2}\right)$. Thus, it is evident that the Jacobi method is not a feasible solution in most practical networks. To be used in practical networks, it is required that the potential-based method converge significantly faster.
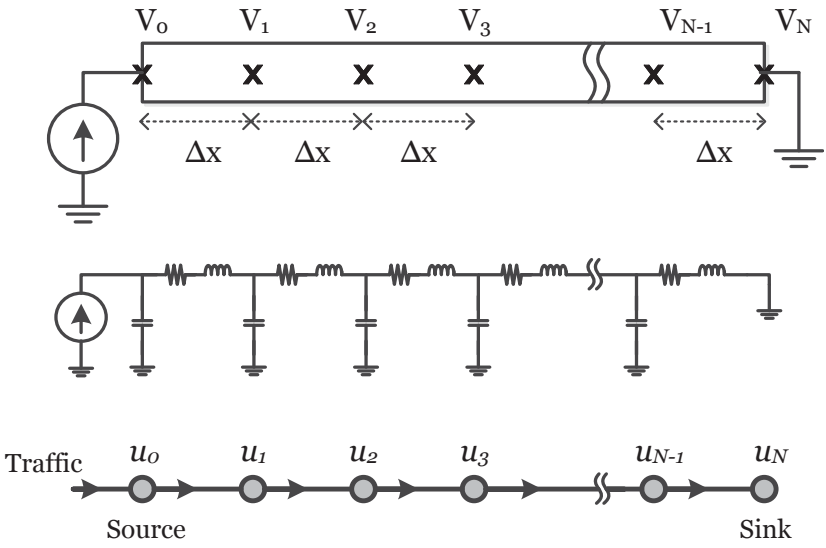

Fig. 2. 1) A continuous single stub transmission line discretized on indicated points $V_{i}=V(i \Delta x)$ with spacing $\left.\Delta x, 2\right)$ Equivalent circuit model consists of series of elementary components, each containing primary elements $R, L, C$, 3) Equivalent network graph model as a path containing $N+1$ nodes with source and sink nodes at both ends.

\section{ITERATIONS INSPIRED BY TRANSMISSION LINES}

In this section, we use a numerical approximation scheme for wave propagation differential equation in transmission lines. Then, we introduce a new iterative method for distributed calculation of potential values in communication networks by drawing analogy between the transient behavior of voltages and currents in transmission line, and potential iterations in a path network. Later in this section, we will explain how the new scheme can be generalized to all network topologies.

\section{A. Numerical Analysis of Wave Propagation in Transmission Lines}

To analyze the transient behavior of voltages and currents, a continuous transmission line can be modeled as a sequence of two-port elementary components, each representing an infinitesimally short segment of transmission line as shown in Figure 2. A pair of first-order partial differential equations, known in classical physics as telegrapher's equations, describes wave propagation in the transmission line

$$
\begin{aligned}
\frac{\partial}{\partial x} I(x, t) & =-C \frac{\partial}{\partial t} V(x, t)-G V(x, t) \\
\frac{\partial}{\partial x} V(x, t) & =-L \frac{\partial}{\partial t} I(x, t)-R I(x, t)
\end{aligned}
$$

where the parameters $R, L, C$ and $G$ are distributed elements of transmission line per unit length known as primary line constants. Inspired by fast transient behavior of voltages and currents, our goal is to model wave propagation as an effective iterative process. An accurate numerical approximation scheme is required to make use of the fast transient behavior. For this purpose, we use the finite difference method (FDM) to discretize telegrapher's equations (4) and to convert them into difference equations by sampling in discrete space. We use $V_{i}^{n}=V(i \Delta x, n \Delta t)$ and $I_{i}^{n}=I(i \Delta x, n \Delta t), i \in$ $\{0,1,2, \ldots, N\}, n \in\{0,1,2, \ldots\}$, to denote the numerical 
approximation for voltages and currents at time sample $n$ and location $i$. In other words, voltages and currents are sampled at discrete points with uniform spacing on both dimensions $(\Delta x, \Delta t)$. Replacing the derivatives in equations (4) by their finite difference approximations results in the following discrete equations:

$$
\begin{aligned}
\frac{I_{i+1}^{n}-I_{i}^{n}}{\Delta x} & \approx-C \frac{V_{i}^{n+1}-V_{i}^{n}}{\Delta t}-G V_{i}^{n} \\
\frac{V_{i+1}^{n+1}-V_{i}^{n+1}}{\Delta x} & \approx-L \frac{I_{i+1}^{n+1}-I_{i+1}^{n}}{\Delta t}-R I_{i+1}^{n+1}
\end{aligned}
$$

Given the initial conditions for voltages and currents along the line, they can both be obtained for every time index $n$. In order to achieve a time balance in (5), the loss terms $G V_{i}^{n}$ and $R I_{i+1}^{n+1}$ can be replaced by their time-averaged version, $\left(V_{i}^{n}+V_{i}^{n+1}\right) / 2$ and $\left(I_{i+1}^{n}+I_{i+1}^{n+1}\right) / 2$, respectively. This also helps get more accurate approximations and reduced discretization error [8]. By applying this change and rearranging the terms in (5), we find the following iterative system for voltages and currents:

$$
\begin{aligned}
V_{i}^{n+1} & =\frac{1-\frac{G \Delta t}{2 C}}{1+\frac{G \Delta t}{2 C}} V_{i}^{n}-\frac{\frac{\Delta t}{C}}{1+\frac{G \Delta t}{2 C}}\left(I_{i+1}^{n}-I_{i}^{n}\right) \\
I_{i+1}^{n+1} & =\frac{1-\frac{R \Delta t}{2 L}}{1+\frac{R \Delta t}{2 L}} I_{i+1}^{n}-\frac{\frac{\Delta t}{L}}{1+\frac{R \Delta t}{2 L}}\left(V_{i+1}^{n+1}-V_{i}^{n+1}\right)
\end{aligned}
$$

where the first equation updates the voltage at the $i$-th node and the second equation updates the current in the $(i+1)$-th branch, as illustrated in Figure 3.

The steady state behavior of voltages and currents in single stub transmission line resembles the final solution for network parameters in a path network as shown in Figure 2, where voltages are analogous to node potentials and so are currents to information flows. Furthermore, the information source and sink nodes are equivalent to current sources and short circuits to ground, respectively. In order to develop a new scheme fully compatible with our desired network behavior, we assume $G$ is zero in our scheme (note that a nonzero $G$ implies leaking information at a node, which violates flow conservation law). However, it is desirable to have a nonzero $R$, because otherwise, we will have a lossless transmission line, which causes instability. This circuit model with zero $G$ and nonzero $R$ leads to steady state response consistent with our desired network behavior, as we will further discuss shortly.

\section{B. General Scheme}

So far, we have used the analogy between a discretized single stub transmission line and a path network. In the next step, we present a general scheme applicable to all possible network topologies. Using the transmission line model representation for a network, nodes and communication links are modeled as capacitors connected to ground and $R L$-series branches, respectively. Unlike the path network where each relay node connects only two communication links, a node in general network topology can be at junction of $M$ branches. However,

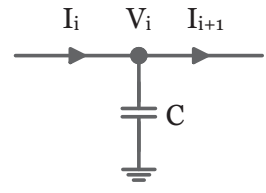

(a)

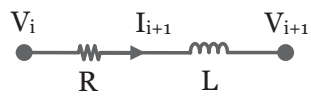

(b)

Fig. 3. The circuit interpretation of iterative equations: (a) the voltage $V$ updated with the difference of input $I_{i}$ and output $I_{i+1}$ currents, (b) the current $I_{i+1}$ updated with the difference of two adjacent node voltages $V_{i}$ and $V_{i+1}$.

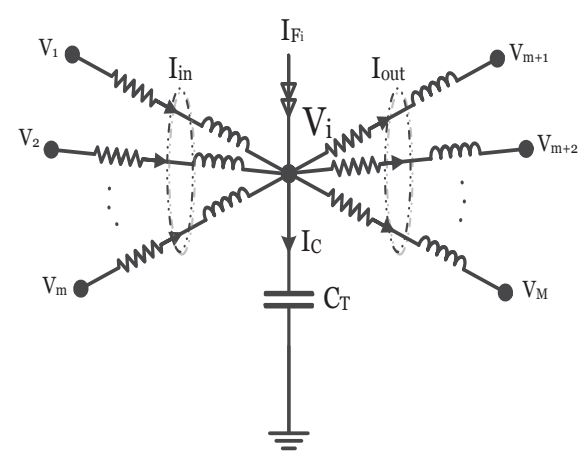

Fig. 4. The Kirchhoff's nodal rule for $i$-th node in general setting as a junction of $M$ branches

there is only one link between two neighboring nodes in both cases. Therefore, the iterative equation (7) for updating currents between two adjacent nodes remains the same as before, while the paired equation (6) for updating voltages at each node requires modification. For the general case, we derive this iterative equation directly from the Kirchhoff's Current Law (nodal rule) as shown in Figure 4. The updating equation for voltage $V_{i}$ can be represented in term of the input current to the node's capacitor, $I_{C_{i}}$, as below:

$$
V_{i}^{n+1}=V_{i}^{n}+\frac{\Delta t}{C_{T}} I_{C_{i}}^{n}
$$

where the product term $I_{C}^{n} \Delta t$ can be interpreted as the amount of new electric charge $\Delta Q^{n}$ deposited in node's capacitor, and if divided by total capacitance $C_{T}$, the voltage difference $\Delta V^{n}$ is obtained. Moreover, according to Figure $4, I_{C_{i}}$ can be calculated as:

$$
I_{C_{i}}^{n}=\sum_{j=1}^{m} I_{j, i}^{n}-\sum_{j=m+1}^{M} I_{i, j}^{n}+I_{F_{i}}^{n}
$$

where the $I_{F_{i}}^{n}$ is the injected current at node $i$, which has a nonzero value for source nodes. The input and output currents are indexed by $j=1, \ldots, m$ and $j=m+1, \ldots, M$, respectively, where $I_{i, j}$ is the current flowing from node $i$ to node $j$. The total capacitance at each node $C_{T}$ is proportional 
to the node degree $M$, which is consistent with the parallel connection of $M$ capacitors.

After designing the iterative process for general network topology, we still need to ensure the stability and convergence to the desired solution. In the next section, we analyze how network parameters affect the stability and rate of convergence.

\section{Stability and Convergence Rate}

In this section, we first choose a path graph as our network configuration to analyze our proposed scheme. Later in this section, we will present a short analysis for general network topologies.

Our iterative scheme for distributed potential calculation can be rewritten in network parameters as follows:

$$
\begin{aligned}
& u_{i}^{n+1}=u_{i}^{n}-\sigma \cdot\left(f_{i+1}^{n}-f_{i}^{n}\right) \\
& f_{i+1}^{n+1}=\alpha \cdot f_{i+1}^{n}-\beta \cdot\left(u_{i+1}^{n+1}-u_{i}^{n+1}\right)
\end{aligned}
$$

where $u_{i}^{n}$ and $f_{i}^{n}, i \in\{0,1, \ldots, N\}, n \in\{0,1,2, \ldots\}$, are node potentials and branch flows at iteration $n$ and location $i$, respectively. Similar to partial differential equations, these coupled difference equations require boundary and initial conditions to lead to a unique solution. For a path network, the start node is assumed to be the traffic source with one unit of traffic rate, and the last node is assumed to be the sink node. The boundary conditions are determined as $f_{0}^{n}=1$ and $u_{N}^{n}=0$ for all $n$. For initial condition, we assume $u_{i}^{0}=0$ for all $i$ and $f_{i}^{0}=0$ for all $i \neq 0$. Applying the flow injection $f_{0}^{n}=1$ to source node, all potentials and flows take values iteratively until finally reaching a steady state value. By carefully choosing network parameters $(\alpha, \beta, \sigma)$ a fast convergence scheme will be achieved.

The pair of equations (10) and (11) can be combined into a single equation for node potentials. By substituting (11) into (10), we find the updated potential as:

$$
\begin{aligned}
u_{i}^{n+1}=u_{i}^{n}-\sigma \cdot & {\left[\left(\alpha \cdot f_{i+1}^{n-1}-\beta \cdot\left(u_{i+1}^{n}-u_{i}^{n}\right)\right)\right.} \\
- & \left.\left(\alpha \cdot f_{i}^{n-1}-\beta \cdot\left(u_{i}^{n}-u_{i-1}^{n}\right)\right)\right]
\end{aligned}
$$

By another substitution of (11) into (12) and rearranging terms, we find the following single equation as the iterations performed at each node:

$$
u_{i}^{n+1}=(\alpha+1) \cdot u_{i}^{n}-\alpha \cdot u_{i}^{n-1}+\beta \sigma \cdot\left(u_{i+1}^{n}-2 u_{i}^{n}+u_{i-1}^{n}\right)
$$

We can rewrite this iterative scheme in more transparent matrix format:

$$
\mathbf{u}^{(n+1)}=2 \mathbf{A} \mathbf{u}^{(n)}-\alpha \mathbf{u}^{(n-1)}+\mathbf{b}^{(n)}
$$

where $\mathbf{u}^{(n)}=\left[u_{0}^{n}, u_{1}^{n}, \ldots, u_{N-1}^{n}\right]^{T}$ is $N \times 1$ potential vector at time $n$ containing node potentials and $\mathbf{b}^{(n)}=[\sigma(1-$ $\alpha), 0, \ldots, 0]^{T}$ is the bias vector containing contributions from boundary conditions. The coefficient matrix $\mathbf{A}$ is a symmetric and tridiagonal matrix defined as

$$
\mathbf{A}=\frac{(\alpha+1)}{2} \mathbf{I}-\frac{\beta \sigma}{2} \mathbf{L}
$$

where $\mathbf{I}$ is $N \times N$ identity matrix, and $\mathbf{L}$ is the Laplacian matrix associated with the path graph.

$$
\mathbf{L}=\left(\begin{array}{cccccc}
1 & -1 & 0 & \ldots & 0 & 0 \\
-1 & 2 & -1 & \ldots & 0 & 0 \\
0 & -1 & 2 & \ldots & 0 & 0 \\
\vdots & \vdots & \ddots & \ddots & \ddots & \vdots \\
0 & 0 & \ldots & -1 & 2 & -1 \\
0 & 0 & \ldots & 0 & -1 & 2
\end{array}\right)
$$

It must be noted that (14) describes a second order iterative scheme, since computing the next state $\mathbf{u}^{(n+1)}$ requires the value of the preceding two states, $\mathbf{u}^{(n)}$ and $\mathbf{u}^{(n-1)}$.

To analyze the stability, the second order iterative scheme can be reduced to first order by defining new vector parameter as follows:

$$
\mathbf{w}^{(n+1)}=\left(\begin{array}{c}
\mathbf{u}^{(n+1)} \\
\mathbf{u}^{(n)}
\end{array}\right)
$$

where $\mathbf{w}^{(n+1)}$ is a column vector of $2 N$ elements. By this definition, the matrix equation (14) is modified to first order iterative format:

$$
\mathbf{w}^{(n+1)}=\mathbf{M w}^{(n)}, \quad \mathbf{M}=\left(\begin{array}{cc}
2 \mathbf{A} & -\alpha \mathbf{I} \\
\mathbf{I} & \mathbf{0}
\end{array}\right)
$$

where $\mathbf{M}$ is $2 N \times 2 N$ matrix. Since each iteration in (18) is a matrix multiplication, stability requires $\left\|\mathbf{M}^{n}\right\|$ be bounded by a constant for large $n$. This condition is satisfied when all eigenvalues of $\mathbf{M}$ are within the unit circle in the complex plane. Therefore, it is required that $\left|\mu_{1}\right| \leq 1$, where $\mu_{1}=\rho(\mathbf{M})$ is the spectral radius of matrix $\mathbf{M}$. This parameter determines the stability and convergence rate of the new scheme. In order to achieve a fast convergent scheme, the gap between spectral radius and unit circle, $d=1-\left|\mu_{1}\right|$, should be maximized. According to (18), $\left|\mu_{1}\right|$ depends on the spectrum of A. Since $\mathbf{A}$ is a diagonalizable matrix, it can be factorized by eigenvalue decomposition to $\mathbf{A}=\mathbf{Q} \mathbf{\Lambda} \mathbf{Q}^{-1}$, where $\mathbf{Q}$ is square matrix with eigenvectors as columns and $\Lambda$ is the diagonal matrix with real eigenvalues on the diagonal, $\Lambda_{i i}=\lambda_{i}$. By substituting eigenvalue decomposition in (18), matrix $\mathbf{M}$ can be factorized to

$$
\mathbf{M}=\left(\begin{array}{cc}
\mathbf{Q} & \mathbf{0} \\
\mathbf{0} & \mathbf{Q}
\end{array}\right)\left(\begin{array}{cc}
2 \boldsymbol{\Lambda} & -\alpha \mathbf{I} \\
\mathbf{I} & \mathbf{0}
\end{array}\right)\left(\begin{array}{cc}
\mathbf{Q}^{-1} & \mathbf{0} \\
\mathbf{0} & \mathbf{Q}^{-1}
\end{array}\right)
$$

Assume $\mu$ is an eigenvalue of $\mathbf{M}$ corresponding to eigenvector $\mathbf{w}=[\mathbf{x}, \mathbf{y}]^{T}$, then according to the above factorization we have

$$
\tilde{\mathbf{M}} \tilde{\mathbf{w}}=\mu \tilde{\mathbf{w}}, \quad \tilde{\mathbf{M}}=\left(\begin{array}{cc}
2 \boldsymbol{\Lambda} & -\alpha \mathbf{I} \\
\mathbf{I} & \mathbf{0}
\end{array}\right)
$$

where $\tilde{\mathbf{w}}=\left[\mathbf{Q}^{-1} \mathbf{x}, \mathbf{Q}^{-1} \mathbf{y}\right]$ is the eigenvector for $\tilde{\mathbf{M}}$ corresponding to the same eigenvalue. Thus, matrix $\tilde{M}$ has the same spectrum as M. Additionally, $\tilde{\mathbf{M}}$ has a special structure (20) such that it can be decomposed to $2 \times 2$ matrices for each eigenvalue $\lambda$

$$
\left(\begin{array}{cc}
2 \lambda & -\alpha \\
1 & 0
\end{array}\right)\left(\begin{array}{l}
x \\
y
\end{array}\right)=\mu\left(\begin{array}{l}
x \\
y
\end{array}\right)
$$


where eigenvalue $\mu$ can be simply computed from the characteristic equation:

$$
\mu^{2}-2 \lambda \mu+\alpha=0
$$

Note that the solution to the above equation is of the form $\mu^{ \pm}=\lambda \pm \sqrt{\lambda^{2}-\alpha}$, where each $\lambda_{i}$ is mapped to a pair of eigenvalues $\mu_{i}^{ \pm}$. To achieve optimum convergence rate, the parameter $\alpha$ should be chosen such that the maximum gap $d_{\max }$ is attained, while satisfying the stability constraint. Assume $\lambda_{1}$ is the spectral radius of matrix $\mathbf{A}$. When $\alpha<\lambda_{1}^{2}$, we have $\left|\mu_{1}\right|>\left|\lambda_{1}\right|$, and therefore, $d<1-\left|\lambda_{1}\right|$. In the other case, when $\alpha \geq \lambda_{1}^{2}$, it will lead to complex eigenvalues, $\mu_{i}^{ \pm}=\lambda_{i} \pm j \sqrt{\alpha-\lambda_{i}^{2}}$. In this case, the gap is equal to $d=1-\sqrt{\alpha} \leq 1-\left|\lambda_{1}\right|$. Thus, the upper bound $d_{\max }$ is achieved by choosing $\alpha$ to be equal to

$$
\alpha_{\text {opt }}=\lambda_{1}^{2}
$$

By choosing the above $\alpha_{\text {opt }}$, all eigenvalues $\lambda_{i}$ are mapped into complex conjugates $\mu_{i}^{ \pm}$on a circle with radius $\sqrt{\alpha}$. Thus, we have $d_{\max }=1-\sqrt{\alpha}$. To further analyze stability, let us define the stability factor parameter, $\xi$, as below:

$$
\xi=\frac{1-\alpha}{1+\alpha}
$$

where this formulation leads to same formulation, $\alpha=\frac{1-\xi}{1+\xi}$. Now we investigate how changing the parameters $(\alpha, \beta, \sigma)$ will affect the spectrum of matrix $\mathbf{A}$. The coefficient matrix in (15) can be rewritten in the new format:

$$
\mathbf{A}=\delta \mathbf{I}+\gamma \mathbf{S}
$$

where the parameters $\delta=\frac{(\alpha+1)}{2}-\beta \sigma$ is translation factor, and $\gamma=\beta \sigma$ is scale factor for the spectrum of square matrix $\mathbf{S}=\mathbf{I}-\frac{1}{2} \mathbf{L}$. These factors $(\delta, \gamma)$ translate and scale the domain of spectrum and affect all eigenvalues

$$
\lambda_{\mathbf{i}}=\delta+\gamma \nu_{\mathbf{i}}, \quad 1 \leq i \leq N
$$

where $\left\{\nu_{i}\right\}$ is the set of eigenvalues of matrix $\mathbf{S}$. For path network topology, the spectrum of $\mathbf{S}$ has the general form [9]:

$$
\nu_{i}=\cos \left(\frac{2 i \pi}{2 N+1}\right), \quad 1 \leq i \leq N
$$

It is obvious that the spectrum of $\mathbf{S}$ is normalized, $\left|\nu_{i}\right| \leq 1$, and the spectral radius is equal to $\rho(\mathbf{S})=\cos \left(\frac{\pi}{2 N+1}\right)$. To satisfy stability constraints, it is necessary that $\left|\lambda_{1}\right| \leq 1$. Moreover, to have the fastest convergence rate it is necessary to minimize $\rho(\mathbf{A})=\left|\lambda_{1}\right|$. As a result, we choose $\delta=0$ so that the spectrum is centered around the origin leading to the optimum scaling factor:

$$
\gamma_{o p t}=\frac{\alpha+1}{2}
$$

Choosing optimum translation and scaling factor will result in spectral radius, $\left|\lambda_{1}\right|=\gamma_{o p t} \cos \left(\frac{\pi}{2 N+1}\right)$. By substituting this result into (23) and simplification of terms, we find the optimum stability factor equal to

$$
\xi_{o p t}=\sin \left(\frac{\pi}{2 N+1}\right)
$$

If the length of the path, $N$, is large enough, we will have the approximation $\xi_{\text {opt }} \approx \frac{\pi}{2 N}$. By substituting this approximation into $d_{\max }=1-\sqrt{\alpha_{\text {opt }}}$, we find the following new approximation for maximum gap as a function of $N$ :

$$
d_{\max } \approx \frac{\pi}{2 N+\pi}
$$

Hence, it can be seen that the rate of convergence for our proposed method is reduced to $O(N)$ iterations, compared to the Jacobi method whose convergence happens by $O\left(N^{2}\right)$ iterations. Therefore, the number of iterations required to converge to the final potential solution decreases significantly, especially when the network size grows.

The analysis for general network topologies is similar to the simple path case. For general case, the nodal equation in (10) is modified to

$$
u_{i}^{n+1}=u_{i}^{n}-\sigma_{i}\left(\sum_{i \rightarrow k} f_{i, k}^{n}-\sum_{j \rightarrow i} f_{j, i}^{n}-f_{b_{i}}^{n}\right)
$$

where $\sigma_{i}=\frac{\sigma}{n_{i}}$ is the network parameter for $i$-th node which is inversely proportional to node degree $n_{i}$. The notations $(i \rightarrow k)$ and $(j \rightarrow i)$ are used to denote the neighbor nodes with output flow from $i$-th node and input flow to $i$-th node, respectively. The flow from node $j$ to node $i$ is denoted by $f_{j, i}$. The paired equation (11) is similar for the general case. By performing same process of substitutions as before, we find a single iterative equation for potentials as follow:

$$
u_{i}^{n+1}=(\alpha+1) u_{i}^{n}-\alpha u_{i}^{n-1}+\beta \sigma_{i}\left(\sum_{j \sim i} u_{j}^{n}-n_{i} u_{i}^{n}\right)
$$

where we used $(j \sim i)$ to denote that node $j$ is neighbor to node $i$. The boundary conditions for the general case is determined by the location of sources and sinks. If the $i$-th node is a source or a sink, the boundary condition appears in the form of $f_{b_{i}}^{n}=$ $c_{i}$ or $u_{i}^{n}=0$ for all times $n$, respectively, where $c_{i}$ is the injected traffic.

Using the normalized Laplacian operator defined as $\Delta u_{i}:=$ $u_{i}-\frac{1}{n_{i}} \sum_{j \sim i} u_{j}^{n}$ in [10], this linear iterative equation can be rewritten in matrix format similar to (14) with coefficient matrix in general form:

$$
\mathbf{A}=\delta \mathbf{I}+\gamma \mathbf{S}, \quad \mathbf{S}=\mathbf{I}-\boldsymbol{\Delta}
$$

where $\boldsymbol{\Delta}=\mathbf{D}^{-1} \mathbf{L}$ is the normalized Laplacian matrix defined in [10], based on Laplacian matrix $\mathbf{L}$ and the degree matrix D. The spectrum of normalized Laplacian matrix of graph is extensively studied in spectral graph theory [11]. The derivation of optimum parameters $\left(\delta_{o p t}, \gamma_{o p t}\right)$ for the general case is similar to the path case. If we name the spectral radius of $\mathbf{S}$ as $\nu_{1}$, then we have $\lambda_{1}=\gamma \nu_{1}$. The optimum stability factor can be computed for the general case:

$$
\xi_{o p t}=\sqrt{1-\nu_{1}^{2}}
$$




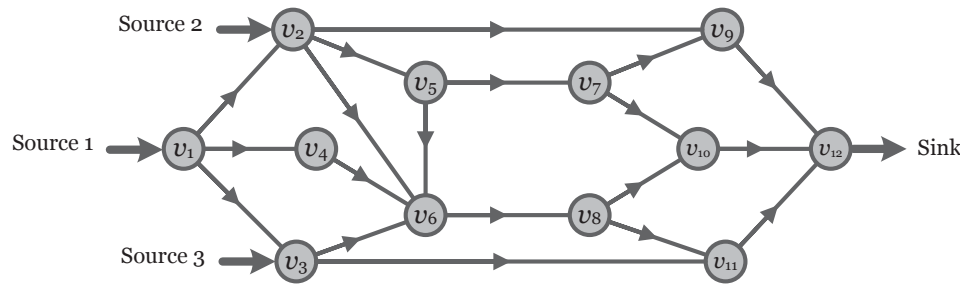

Fig. 5. A typical network with 12 nodes, where the first three nodes $\left(v_{1}, v_{2}, v_{3}\right)$ are source nodes injecting $\left(b_{1}, b_{2}, b_{3}\right)=(1,2,1)$ units of traffic respectively, and node $v_{12}$ is the sink node

\section{Numerical EXAMPLES}

Figure 6 depicts the convergence rate of our proposed method for the path network with $N=20$ compared to the Jacobi method based on averaging. As it can be seen, our proposed method requires considerably smaller number of iterations compared to the Jacobi method. In particular, as the numerical results show, our proposed method requires 70 iterations to converge with error tolerance $2 \%$ compared to 1200 iterations required for Jacobi method. Consider a typical network in Figure 5, in order to evaluate the convergence rate of our proposed method. This network consists of 12 nodes with three nodes $\left(v_{1}, v_{2}, v_{3}\right)$ as source nodes injecting $(1,2,1)$ units of flow, respectively, and $v_{12}$ as sink node gathering all flow from network. In this example, the link capacity is assumed to be the same and equal to one for all links. Figure 7 shows performance of the proposed method in converging to final solution for all node potentials. For the first node potential $v_{1}$, our proposed method requires only 17 iterations to converge with error tolerance $1 \%$ to final solution.

\section{CONCLUSION}

In this paper, we offered a distributed method for calculation of potential values in communication networks. Our method is inspired by wave propagation in a physical transmission line to develop an iterative scheme. The proposed scheme significantly improves the convergence to final potential levels by reducing the number of iterations from $O\left(N^{2}\right)$ to $O(N)$. The calculated potential values can be used in variety of network optimizations such as load balancing, sink placement, and optimal node deployment.

\section{REFERENCES}

[1] M. Kalantari, M. Haghpanahi and M. Shayman, "A p-norm flow optimization problem in dense wireless sensor networks", IEEE INFOCOM, Phoenix, AZ, April 2008.

[2] M. Kalantari and M. Shayman, "Design Optimization of Multi-sink Sensor Networks by Analogy to Electrostatic Theory", IEEE Wireless Communications and Networking Conference, Las Vegas, Nevada, April 2006.

[3] M. Kalantari and M. Shayman, A steepest descent relocation algorithm for placement of sinks in a sensor network, IEEE Globecom, Washington DC, November 2007.

[4] S. Toumpis and L. Tassiulas, Optimal deployment of large wireless sensor networks", IEEE Transactions on Information Theory, vol. 52, no. 7, pp. 29352953, 2006.

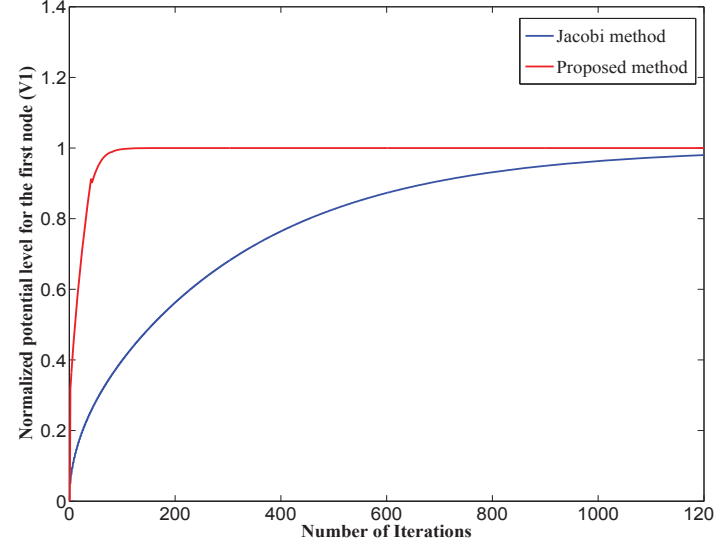

Fig. 6. Convergence rate comparison of potential levels for path network with $N=20$ links and source and sink node at both end for two different iterative techniques: 1)Jacobi method with averaging policy, 2) our proposed method with optimum stability factor $\left(\xi=\frac{\pi}{40}\right)$

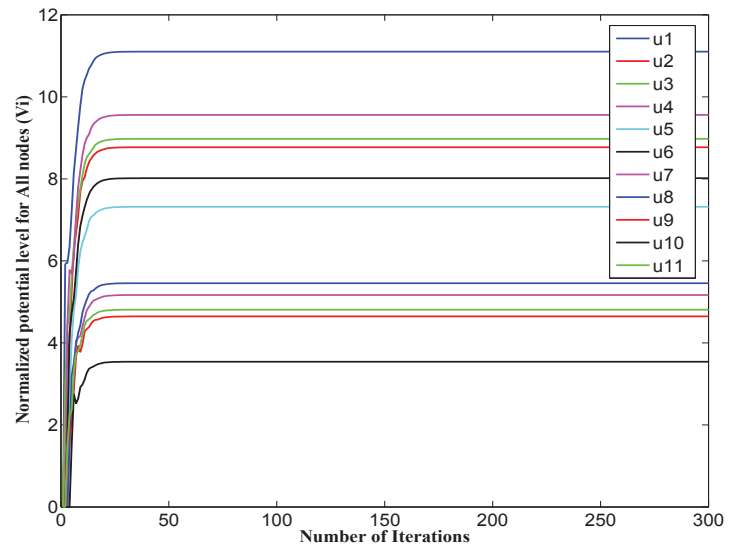

Fig. 7. The convergence rate performance of proposed method for all node potentials

[5] S. Anaraki, "Distributed flow optimization in dense wireless networks", University of Maryland M.Sc. Thesis, Available online at: http : //ece.umd.edu/anaraki/ms_thesis.pdf, 2011.

[6] S. Z. Anaraki, M. Kalantari, "Filling gap between discrete and continuous space flow models in dense wireless networks", 2011 IEEE International Conference on Communications, IEEE, 2011.

[7] S. Z. Anaraki, M. Kalantari, "Acceleration of distributed minimax flow optimization in networks", 2011 45th Annual Conference on Information Sciences and Systems (CISS), IEEE, 2011.

[8] J. B. Schneider, "Understanding the Finite-Difference Time-Domain Method", Available at: www.eecs.wsu.edu/schneidj/ufdtd, 2010.

[9] L. Lovász, J. Pelikan, "On the eigenvalues of trees", Period. Math. Hung., vol. 3, no. 1, pp. $175182,1973$.

[10] A. Banerjee, J. Jost, "On the spectrum of the normalized graph Laplacian", Linear Algebra and its Applications, 428(1112):30153022, 2008.

[11] F. Chung, "Spectral graph theory", AMS, vol. 92, 1997. 